\title{
Mapping Genomic Scaffolds to Chromosomes Using Laser Capture Microdissection in Application to Hawaiian Picture-Winged Drosophila
}

\author{
Lin Kang $^{a} \quad$ Phillip George $^{b}$ Donald K. Price Igor Sharakhov $^{b, f}$ \\ Pawel Michalak ${ }^{\mathrm{a}, \mathrm{c}} \mathrm{d}$ \\ ${ }^{a}$ Biocomplexity Institute and b Department of Entomology, Virginia Tech, ${ }^{\mathrm{C}}$ Edward Via College of Osteopathic \\ Medicine, and ${ }^{\mathrm{d} C e n t e r}$ for Molecular Medicine and Infectious Diseases, Virginia-Maryland College of Veterinary \\ Medicine, Blacksburg, VA, and ' School of Life Sciences, University of Nevada Las Vegas, Las Vegas, NV, USA; \\ f Laboratory of Ecology, Genetics and Environmental Protection, Tomsk State University, Tomsk, Russia
}

\section{Keywords}

Chromosome mapping · Hawaiian Drosophila $\cdot$ Laser capture $\cdot$ Microdissection

\begin{abstract}
Next-generation sequencing technologies have led to a decreased cost and an increased throughput in genome sequencing. Yet, many genome assemblies based on short sequencing reads have been assembled only to the scaffold level due to the lack of sufficient chromosome mapping information. Traditional ways of mapping scaffolds to chromosomes require a large amount of laboratory work and time to generate genetic and/or physical maps. To address this problem, we conducted a rapid technique which uses laser capture microdissection and enables mapping scaffolds of de novo genome assemblies directly to chromosomes in Hawaiian picture-winged Drosophila. We isolated and sequenced intact chromosome arms from larvae of $D$. differens. By mapping the reads of each chromosome to the recently assembled scaffolds from 3 Hawaiian picturewinged Drosophila species, at least $67 \%$ of the scaffolds were
\end{abstract}

\section{KARGER}

(C) 2017 S. Karger AG, Basel

E-Mail karger@karger.com

www.karger.com/cgr successfully assigned to chromosome arms. Even though the scaffolds are not ordered within a chromosome, the fastgenerated chromosome information allows for chromosome-related analyses after genome assembling. We utilize this new information to test the faster- $X$ evolution effect for the first time in these Hawaiian picture-winged Drosophila species.

(c) 2017 S. Karger AG, Basel

Along with the rapid development of massively parallel sequencing, or next-generation sequencing (NGS), DNA sequencing capabilities grow exponentially. This allows researchers to perform de novo genome assemblies for thousands of species, from viruses and insects to mammals (e.g., Genome 10K, i5K [Genome 10K Community of Scientists, 2009; i5K Consortium, 2013]). However, the complexity of the assembly task using short reads still poses a major challenge. Genome assemblies are hierarchical, and a typical de novo assembly process, especially for large eukaryotic genomes, often ends at the level of scaffolds, assembled from shorter as-

Pawel Michalak

Biocomplexity Institute

Virginia Tech

Blacksburg, VA 24061 (USA)

E-Mail pmichalak@vcom.vt.edu 
sembly components called contigs (Ensembl database, http://www.ensembl.org/). With sufficient mapping information, scaffolds can be assembled into chromosomes. The mapping information may include genetic, physical, or optical maps [Dean et al., 2005; Chamala et al., 2013; Dong et al., 2013], as well as sequence data from long insert size or mate pair reads. All of these are highly laborious tasks even before the genomic location can be obtained.

An alternative approach for mapping scaffolds to chromosomes can be synteny-based, where a closely related species with assembled chromosome information is used as reference for mapping [Drososphila 12 Genomes Consortium, 2007; Stark et al., 2007; Schaeffer et al., 2008; Kim et al., 2013], or synteny across species inferred from a comparative analysis is utilized to order the scaffolds [Husemann and Stoye, 2010; Aganezov et al., 2015]. However, chromosome synteny alone should be treated with caution since structural and chromosomal rearrangements may have occurred even between closely related species. Assembly errors from the reference species assembly may also be transferred to the new species assembly [Ekblom and Wolf, 2014], thus affecting the accuracy of the mapping. For example, Drosophila melanogaster and D. simulans have diverged from each other for 2-8 million years [Obbard et al., 2012], but they have highly collinear genomes, with the exception of a large fixed inversion in chromosome arm 3R [Sturtevant and Plunkett, 1926] and a few minor inversions in the other chromosomes [reviewed in Aulard et al., 2004]. Yet, unlike $D$. melanogaster, $D$. simulans has very few segregating inversions, which provides certain advantages for the analysis of natural variation and experimental studies [Aulard et al., 2004; Schlötterer et al., 2015].

Alternatively, relatively quick chromosome mapping information can be obtained through the isolation of euchromatic segments from distinct chromosome arms using laser capture microdissection (LCM), coupled with whole-genome amplification and NGS [George et al., 2014]. Here, we test this approach in application to polytene chromosomes of Hawaiian picture-winged Drosophila species, an iconic example of sequential colonization, speciation, and adaptive radiation [Carson and Clague, 1995; Carson, 1997]. The Hawaiian picture-winged Drosophila played an important role as a model system in substantiating the modern evolutionary synthesis and the biological species concept [Mayr, 1963, 1982] and the formulation of the founder-event model for speciation [Carson and Templeton, 1984]. The Hawaiian Drosophila are estimated to have colonized the Hawaiian Islands ap-

LCM for Mapping Genomic Scaffolds to Drosophila Chromosomes proximately 25 mya and radiated into up to 800 extant species with approximately 120 of these belonging to the large picture-wing group [O'Grady et al., 2011]. Speciation in the Hawaiian picture-winged Drosophila involved allopatric speciation following the "progression rule" that occurred when a new species was founded on each new island as it emerged from the volcanic hotspot in the middle of the Pacific plate [Carson and Clague, 1995]. Sympatric species divergence within islands also appears to have occurred, in part due to switching host-plant associations [Magnacca and Price, 2015]. More recently, many of the Hawaiian picture-wing Drosophila have declined in numbers with 11 species now classified as endangered, including $D$. differens and $D$. heteroneura. However, to date genome information for the Hawaiian Drosophila is limited. Only 1 species of Hawaiian picture-wing Drosophila, D. grimshawi, was sequenced by the Drosophila $12 \mathrm{Ge}-$ nomes Consortium [2007], but this genome remains unfinished, and the scaffolding was partly guided by synteny with distantly related species. We have recently sequenced, pre-assembled, and analyzed genomes of 3 other Hawaiian picture-winged Drosophila species [Kang et al., 2016]. Chromosomal variation in Hawaiian picture-winged Drosophila was of great taxonomical and biogeographical value, as the polytene karyotypes had been arranged into lineages reflecting a series of inversions within chromosome arms and species divergence [Carson, 1983]. Apart from the inversions, all these species possess 6 pairs $(2 n=12)$ of highly syntenic chromosomes.

Based on LCM and NGS from polytene chromosomes, we assigned scaffolds to chromosomes from the following 3 closely related species with pre-assembled genomes: D. silvestris, D. heteroneura, and D. planitibia [Kang et al., 2016]. Although these scaffolds are still not localized or ordered within a chromosome, the initial chromosome information can be useful for further mapping, as well as for certain population and evolutionary analyses. For the latter, we exemplify the utility of LCM-based mapping in application to a preliminary test for faster-X chromosome evolution [Charlesworth et al., 1987] among the Hawaiian picture-winged Drosophila.

\section{Materials and Methods}

Flies and Genome Assemblies

D. differens is endemic to the island of Molokai, and the individuals used in this study are from the stock \# Z27921 that was obtained from the Hawaiian Drosophila stock center at the University of Hawaii at Manoa (courtesy of K. Kaneshiro). The genome assemblies of $D$. silvestris, D. heteroneura, and D. planitibia 
Table 1. Quality control for mapping reads against Hawaiian Drosophila species and human genome references

\begin{tabular}{|c|c|c|c|c|c|c|c|c|}
\hline \multirow[t]{2}{*}{ Sample ID } & \multirow[t]{2}{*}{ Reads, $n$} & \multirow[t]{2}{*}{ Total bases } & \multirow[t]{2}{*}{ Q20, \% } & \multirow[t]{2}{*}{ Q30, \% } & \multirow[t]{2}{*}{ GC content, $\%$} & \multicolumn{3}{|c|}{ Mapping rate, $\%$} \\
\hline & & & & & & D. planitibia & D. grimshawi & human \\
\hline $2^{\mathrm{a}}$ & $8,643,203$ & $1,288,795,937$ & 97.05 & 95.04 & 44.21 & 14.31 & 4.42 & 68.26 \\
\hline $3^{\mathrm{a}}$ & $9,965,841$ & $1,289,067,003$ & 97.60 & 95.91 & 45.75 & 18.94 & 8.91 & 85.98 \\
\hline 5 & $10,098,983$ & $1,361,545,667$ & 97.32 & 95.43 & 44.77 & 47.68 & 39.30 & 59.15 \\
\hline $6^{\mathrm{a}}$ & $10,968,595$ & $1,484,765,509$ & 97.36 & 95.49 & 44.95 & 20.48 & 12.40 & 60.74 \\
\hline 7 & $10,225,920$ & $1,255,423,051$ & 97.66 & 95.94 & 45.02 & 65.61 & 60.82 & 30.09 \\
\hline 9 & $9,429,412$ & $1,238,139,403$ & 97.43 & 95.55 & 44.75 & 66.36 & 63.26 & 22.90 \\
\hline 10 & $8,843,571$ & $1,172,290,243$ & 97.46 & 95.65 & 44.58 & 50.27 & 44.05 & 46.23 \\
\hline 11 & $10,677,556$ & $1,334,570,886$ & 97.71 & 96.01 & 44.83 & 70.56 & 67.22 & 20.96 \\
\hline 12 & $9,846,903$ & $1,270,347,221$ & 97.48 & 95.66 & 44.64 & 69.53 & 65.44 & 31.37 \\
\hline 13 & $11,632,471$ & $1,626,606,120$ & 97.29 & 95.32 & 43.80 & 94.76 & 92.75 & 17.29 \\
\hline 14 & $10,494,444$ & $1,393,026,007$ & 97.49 & 95.68 & 44.57 & 66.80 & 62.39 & 30.80 \\
\hline 15 & $12,056,935$ & $1,506,469,114$ & 97.46 & 95.59 & 45.39 & 79.14 & 74.65 & 25.88 \\
\hline 16 & $10,244,193$ & $1,303,887,668$ & 97.50 & 95.66 & 44.71 & 86.22 & 84.03 & 19.49 \\
\hline 17 & $10,747,167$ & $1,403,344,374$ & 97.58 & 95.80 & 44.00 & 76.73 & 73.18 & 21.06 \\
\hline 19 & $10,866,170$ & $1,444,046,227$ & 97.40 & 95.53 & 44.10 & 70.38 & 65.89 & 29.37 \\
\hline 20 & $9,957,740$ & $1,284,186,408$ & 97.58 & 95.76 & 44.45 & 90.78 & 88.51 & 17.38 \\
\hline $21^{\mathrm{a}}$ & $13,352,093$ & $2,076,865,360$ & 96.97 & 94.81 & 40.66 & 4.25 & 4.22 & 6.30 \\
\hline 22 & $14,159,655$ & $1,918,046,964$ & 97.32 & 95.41 & 44.29 & 62.48 & 57.04 & 36.67 \\
\hline $23^{\mathrm{a}}$ & $9,501,479$ & $1,430,814,118$ & 97.03 & 95.01 & 44.01 & 28.27 & 18.53 & 69.33 \\
\hline 24 & $10,323,650$ & $1,487,264,963$ & 97.30 & 95.35 & 45.45 & 46.02 & 41.10 & 33.83 \\
\hline $25^{\mathrm{a}}$ & $9,922,001$ & $1,355,634,235$ & 97.47 & 95.65 & 45.58 & 14.68 & 7.80 & 51.13 \\
\hline $26^{\mathrm{a}}$ & $8,751,880$ & $1,339,402,154$ & 97.04 & 95.03 & 44.19 & 22.83 & 12.27 & 73.06 \\
\hline 27 & $8,855,911$ & $1,246,340,475$ & 97.14 & 95.15 & 44.31 & 53.86 & 46.03 & 50.66 \\
\hline 28 & $10,279,633$ & $1,230,529,432$ & 97.72 & 96.02 & 44.84 & 50.88 & 46.16 & 31.02 \\
\hline
\end{tabular}

${ }^{\text {a }}$ Filtered samples.
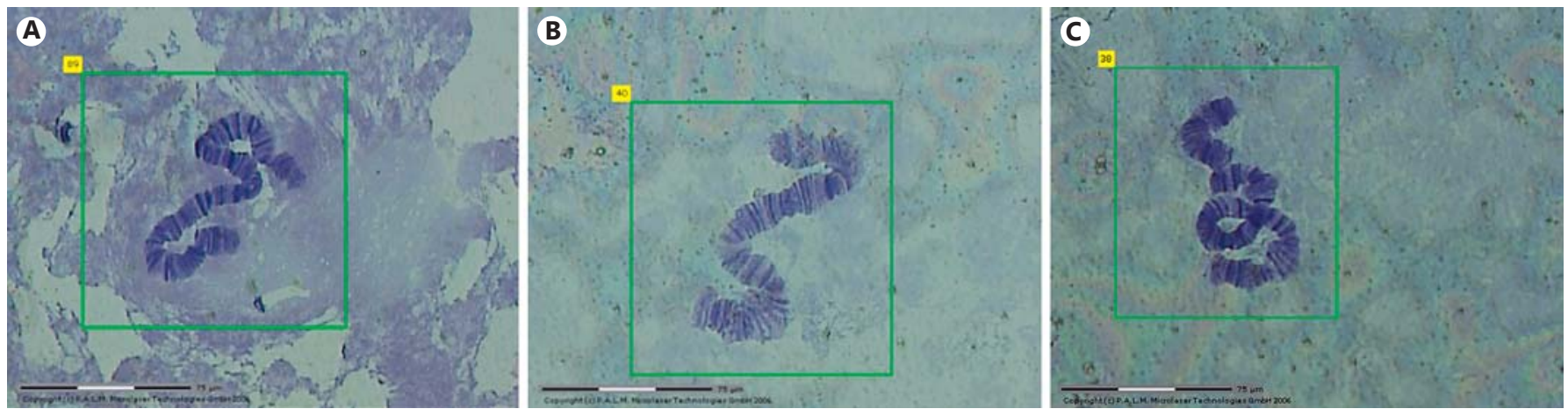

Fig. 1. A-C Examples of microscopic images of LCM Drosophila differens chromosomes.

were obtained as described elsewhere [Kang et al., 2016]. Genes and repeats in the genome sequence were initially annotated as described previously [Kang et al., 2016] (see also below).

Polytene Chromosome Preparation, LCM, and Sequencing

Intact chromosome arms were isolated from larvae of $D$. differens using a published LCM protocol for isolating and amplifying the euchromatic segments of individual polytene chromosome arms [George et al., 2014]. One pair of salivary glands was placed in 1 drop of Carnoy solution on a Zeiss 1.0 PET membrane slide. Salivary glands were split into approximately $2-4$ sections with dissecting needles and placed into a drop of $50 \%$ propionic acid on clean slides under a dissection microscope. A drop of $50 \%$ propionic acid was added to the glands for 3-5 min at room temperature. A siliconized coverslip was placed on top of the droplet. The slide was covered with an absorbent material (filter paper was used 
for this method), and while using the eraser side of a pencil, a generous amount of pressure was applied to the coverslip by tapping on it repeatedly with the eraser. The slides were heated to $60^{\circ} \mathrm{C}$ on a slide denaturation/hybridization system for 15-20 min to aid in flattening the polytene chromosomes. The slides were put into a humid chamber at $4^{\circ} \mathrm{C}$ overnight to allow the acid to further flatten the chromosomes. Then, they were placed in cold 50\% ethanol for $10 \mathrm{~min}$ and the coverslips were gently removed. Slides were dehydrated in 50, 70, 90, and 100\% ethanol for 5 min each and air dried. A solution of GURR buffer was prepared by adding a single buffer tablet to $1 \mathrm{~L}$ of distilled water and autoclaving. The Giemsa solution was prepared by adding $1 \mathrm{~mL}$ of Giemsa staining solution to $50 \mathrm{~mL}$ of GURR buffer. Air-dried slides were placed in Giemsa solution for $10 \mathrm{~min}$ and washed 3 times in $1 \times$ PBS. Slides were dried again in a controlled sterile climate to avoid contamination. LCM of chromosomes was done with the PALM MicroBeam Laser Microdissection system and the PALMRobo software as described previously [George et al., 2014]. Microdissected chromosomes were lysed to release gDNA. Whole-genome amplification was used to amplify the DNA from the microdissected material and to create libraries with the PicoPLEX ${ }^{\mathrm{TM}}$ DNA-seq kit (Rubicon Genomics, Ann Arbor, MI, USA). The 250-bp single-end libraries were further amplified exponentially with primers containing unique Illumina dual barcodes suitable for Illumina Miseq. A total of 24 single-chromosome samples were generated (Fig. 1). Each sample was assigned a putative chromosome ID according to its karyotype/chromosome banding pattern [Carson, 1983].

\section{Quality Control and Mapping}

Raw reads from sequencing were quality-controlled and filtered through FastqMcf [Aronesty, 2013]. Briefly, adaptors were clipped, and reads with average quality $<30$ or with more than $5 \mathrm{~N}$ (bad) bases were discarded. To reduce the possible contamination, reads were mapped to the bacteria database downloaded from NCBI (http://www.ncbi.nlm.nih.gov/), and unmapped reads were kept. To assess the quality of the samples, the clean reads were next mapped to D. planitibia [Kang et al., 2016], the D. grimshawi genome reference (http://flybase.org/), and the human genome reference (hg19; http://genome.ucsc.edu/) using BWA [Li and Durbin, 2009] with default parameters (Table 1). Samples with reads mapping against the human reference at a rate $>60 \%$ or mapping against the $D$. planitibia reference at a rate $<40 \%$ were removed ( 7 samples). The thresholds were found through trial and error to optimize the number of samples used. To obtain more valid chromosome information, reads from the remaining 17 samples were then mapped to repeat-masked $D$. melanogaster reference $(\mathrm{dm} 3)$. The sizes of regions with at least 5 reads mapped were counted for each $D$. melanogaster chromosome. For each sample, the ratio between the highest count and the second highest count was calculated. Samples with this ratio $<10$ were considered possible chromosome cross-contaminations and were thus discarded ( 5 samples; Table 2). The remaining 12 samples were then merged into 5 groups with samples sharing the same best chromosome hit against D. melanogaster, and synteny with Muller element arm (for D. melanogaster, chrX, chr2L, chr2R, chr3L, chr3R, and chr4 correspond to Muller elements $\mathrm{A}, \mathrm{B}, \mathrm{C}, \mathrm{D}, \mathrm{E}$, and F, respectively).

\section{Scaffold Chromosome Annotation}

Reads of samples within the same group were combined to maximize data utilization. Grouped reads were mapped to draft
Table 2. Counts of mapping for each sample against masked D. melanogaster genome reference

\begin{tabular}{|c|c|c|c|c|c|c|}
\hline \multirow[t]{2}{*}{ ID } & \multicolumn{2}{|l|}{ Best } & \multicolumn{2}{|c|}{ Second best } & \multirow[t]{2}{*}{ Ratio $^{a}$} & \multirow[t]{2}{*}{ Group } \\
\hline & $\begin{array}{l}\text { chromo- } \\
\text { some }\end{array}$ & count & $\begin{array}{l}\text { chromo- } \\
\text { some }\end{array}$ & count & & \\
\hline 5 & chr2R & 207,475 & chr3R & 1,482 & 140.00 & MullerC \\
\hline 12 & chr2R & 255,165 & $\operatorname{chr} 2 \mathrm{~L}$ & 1,139 & 224.03 & \\
\hline 16 & chr2R & 286,372 & $\operatorname{chr} 2 \mathrm{~L}$ & 1,073 & 266.89 & \\
\hline 28 & chr2R & 129,207 & chr3R & 2,105 & 61.38 & \\
\hline 7 & chr3L & 364,033 & chr3R & 1,128 & 322.72 & MullerD \\
\hline 10 & chr3L & 212,133 & chr3R & 767 & 276.57 & \\
\hline 11 & chr3L & 332,434 & $\operatorname{chr} 2 \mathrm{~L}$ & 2,182 & 152.35 & \\
\hline $14^{\mathrm{b}}$ & $\operatorname{chr} 3 \mathrm{~L}$ & 382,687 & chr3R & 87,431 & 4.38 & \\
\hline 9 & $\operatorname{chr} 2 \mathrm{~L}$ & 231,771 & chr3R & 836 & 277.24 & MullerB \\
\hline 19 & $\operatorname{chr} 2 \mathrm{~L}$ & 395,533 & $\operatorname{chr} 3 \mathrm{~L}$ & 9,116 & 43.39 & \\
\hline 15 & chrX & 251,583 & chr2R & 1,463 & 171.96 & MullerA \\
\hline 27 & $\operatorname{chrX}$ & 225,643 & $\operatorname{chr} 2 \mathrm{~L}$ & 937 & 240.81 & \\
\hline 20 & chr3R & 416,570 & $\operatorname{chrX}$ & 3,482 & 119.64 & MullerE \\
\hline $13^{\mathrm{b}}$ & chr3R & 284,731 & $\operatorname{chr} 2 \mathrm{~L}$ & 237,464 & 1.20 & \\
\hline $17^{\mathrm{b}}$ & chr3R & 188,318 & $\operatorname{chr} 2 \mathrm{~L}$ & 112,463 & 1.67 & \\
\hline $22^{\mathrm{b}}$ & $\operatorname{chr} 2 \mathrm{~L}$ & 191,315 & chr3R & 128,616 & 1.49 & \\
\hline $24^{\mathrm{b}}$ & chr2R & 95,415 & chr2L & 51,737 & 1.84 & \\
\hline
\end{tabular}

${ }^{a}$ Count of the best divided by count of the second best. ${ }^{b}$ Discarded samples.

Table 3. Summary of scaffold group information assignment for Hawaiian Drosophila species

\begin{tabular}{llll}
\hline Species & $\begin{array}{l}\text { Size of assembly, } \\
\mathrm{Mb}\end{array}$ & $\begin{array}{l}\text { Size of assigned } \\
\text { scaffolds, Mb }\end{array}$ & Percent \\
\hline D. silvestris & 145.88 & 122.85 & 84.21 \\
D. heteroneura & 146.62 & 122.94 & 83.85 \\
D. planitibia & 188.99 & 129.39 & 68.46 \\
D. grimshawi & 207.72 & 144.76 & 69.69 \\
\hline
\end{tabular}

assemblies from 3 Drosophila species (D. silvestris, D. heteroneu$r a, D$. planitibia) using BWA with default parameters, while the draft assemblies were repeat-masked before mapping by RepeatMasker (Open-4.0, http://www.repeatmasker.org). For each scaffold in an assembly, mapping information for all 5 groups was collected, and the lengths of regions covered by at least 5 reads from each group were counted. Scaffold was assigned with chromosome/group information if the largest length was $>1,000$ and the second largest length was less than one tenth of the largest one (e.g., scaffold 10 has a 10,000-bp region with each base covered by at least 5 reads from group MullerA, and it also has a 500-bp region covered by at least 5 reads from group MullerB; thus, we assigned MullerA to scaffold10). The remaining scaffolds were labeled as unassigned. In general, more than $65 \%$ of the total size of scaffolds could be assigned with group information for each species (Table 3 ). 
Fig. 2. Mapping of reads from LCM samples against Drosophila melanogaster chromosomes.

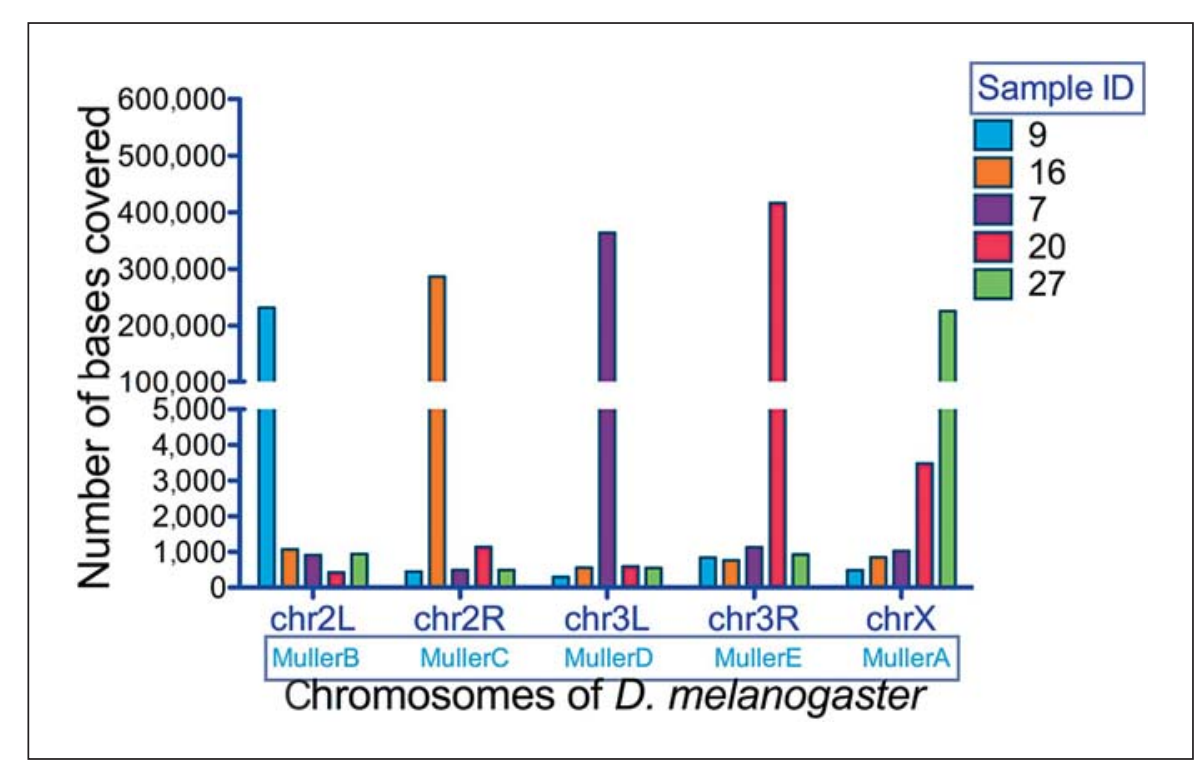

\section{Gene Prediction and Annotation}

Protein-coding genes were predicted using MAKER2 [Holt and Yandell, 2011], which used D. melanogaster protein sequences from FlyBase (r6.02, http://flybase.org) as protein homology evidence, and integrated with prediction methods including BLASTX, SNAP [Korf, 2004], and AUGUSTUS [Stanke and Waack, 2003]. In order to further increase the accuracy of gene prediction, we included the RNA-seq data to the prediction process. RNA-seq data for D. silvestris, D. heteroneura, and D. planitibia were downloaded from NCBI Gene Expression Omnibus (GEO, www.ncbi. nlm.nih.gov/geo) under accession number GSE80124 and from our previous study [Brill et al., 2016]. Adapters were removed from the raw sequencing reads. Duplicated and low-quality reads were discarded using FastqMcf [Aronesty, 2013] with default parameters. To minimize possible contamination, reads were aligned to the NCBI bacterial database, and only unmapped reads were kept to assemble the transcriptome. The processed reads from each species were merged together and assembled with Trinity [Grabherr et al., 2011] (with parameter "-trimmomatic"). The assembled RNA-seq data were input to the MAKER2 pipeline as EST evidence. Predicted genes were subsequently used as query sequences in a blastx database search of NR database (non-redundant database, http://www.ncbi.nlm.nih.gov/). Blastx alignments with evalue greater than $1 \mathrm{e}^{-10}$ were discarded, and the top hit (or top hit from Drosophila species if existed) was used to annotate the query genes.

\section{Genome Completeness}

Two methods were used for genome completeness estimation. CEGMA [Parra et al., 2007] examines the existence of 248 core eukaryotic genes in the assembly. BUSCO [Simão et al., 2015] was used to assess universal single-copy orthologs of eukaryotes in the assembly.

\section{$\mathrm{Ka} /$ Ks Ratio}

In order to reduce the possible impact of gene mis-annotations on the $\mathrm{Ka} / \mathrm{Ks}$ (or $\mathrm{dN} / \mathrm{dS}$ ) ratio, we used only annotations against
Swissprot (http://www.ebi.ac.uk/uniprot). Blastx alignments with e-values greater than $1 \mathrm{e}^{-40}$ or identity $<40 \%$ were discarded. D. grimshawi was used as an outgroup, sequences with the same annotation were grouped together, and Clustal-omega [Sievers et al., 2011] was used to conduct the multiple sequence alignments. Nucleotide sequences were parsed to amino acid sequences before carrying out multiple-sequence alignments to avoid possible frameshifts, and the amino acid sequences of the alignment were changed back to nucleotide sequences for $\mathrm{Ka} / \mathrm{Ks}$ calculations. PAML [Yang, 2007] (version 4.7) was used to calculate the pairwise $\mathrm{Ka} / \mathrm{Ks}$ ratio values, setting the model $=0$ in the control file of codeml. To further minimize the possible effect of wrong annotations and grouping, Ks values $>2$ were excluded from further analyses, and the maximal $\mathrm{Ka} / \mathrm{Ks}$ value was set to be 3 . Models M7/M8 along with likelihood ratio tests were applied to test for the significance of positive selection, with $p$ values generated from $\chi^{2}$ distribution [Nielsen and Yang, 1998].

\section{Results}

\section{Genome Completeness and Annotation}

The pre-assembled genomes were assessed for their completeness with 2 methods, CEGMA [Parra et al., 2007] and BUSCO [Simão et al., 2015] (online suppl. Tables 1, 2; see www.karger.com/doi/10.1159/000481790 for all online suppl. material). The number of contigs for the 3 assemblies ranged from 6,692 (D. silvestris) to 15,471 (D. planitibia), with the average completeness of $95.43 \%$ and $89.51 \%$ by CEGMA and BUSCO, respectively. Among the 3 species, D. planitibia had the largest assembled genome size of $189 \mathrm{Mb}$, while $D$. silvestris had the smallest assembled genome size of $146 \mathrm{Mb}$. The numbers 


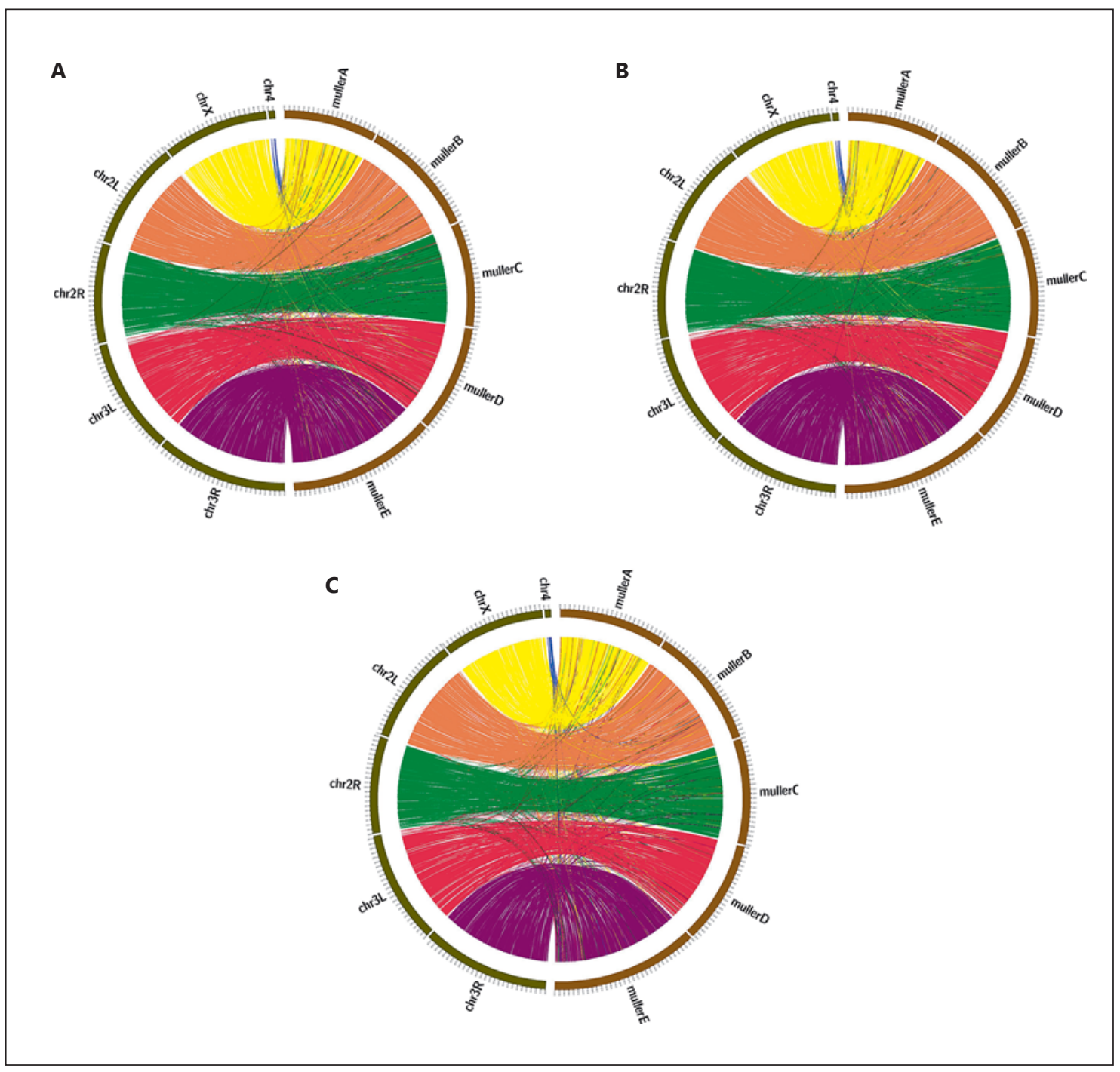

Fig. 3. Circos diagrams showing genomic synteny of Hawaiian Drosophila with D. melanogaster. Links were created based on the alignment results between D. heteroneura (A), D. silvestris (B), and D. planitibia (C) and D. melanogaster (reference in $\mathbf{A}-\mathbf{C}$ ) using Blastn. Alignments spanning less than 100 bp were discarded.

of predicted protein-coding genes ranged between 10,919 (D. heteroneura) and 12,746 (D. planitibia), correlating with their genome sizes (online suppl. Table 3). More than $97 \%$ of the predicted genes could be annotated against the non-redundant protein database.

\section{Chromosome Isolation and Sequencing}

A total of 24 chromosome samples from 3rd instar larvae of $D$. differens, an endemic species from Molokai (sister species to D. planitibia from Maui), were isolated and sequenced (Fig. 1). An average of 10,410,225 reads or $1,405,892,022 \mathrm{bp}$ were generated for each sample. After a

LCM for Mapping Genomic Scaffolds to Drosophila Chromosomes stringent quality control, including tests for interchromosomal contamination, as well as foreign DNA contamination, only 12 samples were used for chromosome mapping and further analysis (Table 2; see Materials and Methods). The majority of reads from each of the 12 samples were mapped to a single Muller element using D. melanogaster as a reference, with the ratio of the highest count and the second highest count $>10$ (Fig. 2; Table 2).

\section{Mapping Scaffolds to Chromosomes}

The 12 samples were then merged into 5 groups corresponding to Muller elements, based on chromosomal 


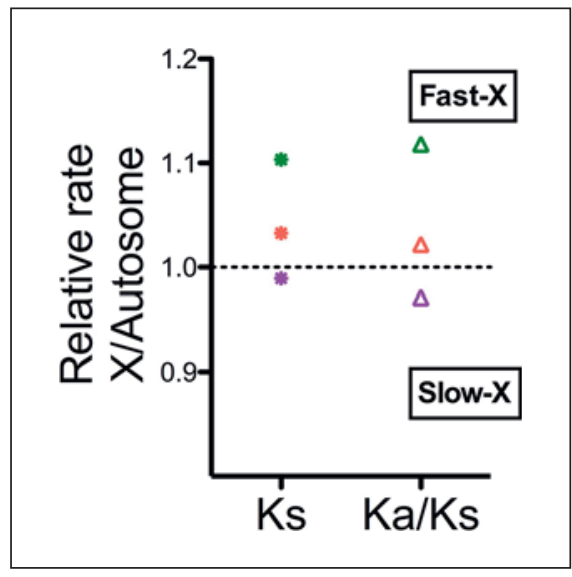

Fig. 4. Relative rates of $\mathrm{Ks}$ and $\mathrm{Ka} / \mathrm{Ks}$ between autosomal genes and $\mathrm{X}$-linked genes. Ks is indicated by asterisks, $\mathrm{Ka} / \mathrm{Ks}$ by triangles with Drosophila heteroneura rates in purple, D. silvestris in red, and $D$. planitibia in green.

synteny. Reads from each group were merged and mapped against each assembly from each species separately. Scaffolds from each assembly were assigned a Muller element label if stringency criteria were met (see Materials and Methods). More than 68\% (68.46-84.21\%) of the scaffolds were successfully assigned with Muller element labels (Table 3). Merging scaffolds into LCM-derived chromosomes according to their Muller element labels, and mapping the chromosomes from each species against the D. melanogaster chromosomes generated a general chromosomal alignment (Fig. 3). A large proportion of each LCM-derived chromosome successfully mapped to a single $D$. melanogaster chromosome, confirming the validity of the method, as well as high chromosomal synteny between Hawaiian Drosophila and D. melanogaster. "Aberrant" alignments across chromosomes likely indicate chromosomal and structural rearrangement events after the split from the common ancestor with $D$. melanogaster. Interchromosomal contamination cannot be completely ruled out as a source of the pattern of spurious structural rearrangements, but we note that DNA contamination tends to be magnified through whole-genome amplification and is thus relatively easy to detect and eliminate at the sample level.

\section{A Preliminary Test for Faster-X Chromosome \\ Evolution}

Even though the scaffolds within LCM-derived chromosomes were not ordered, information on approximate chromosome mapping is useful for addressing a variety of questions, such as those related to sex chromosomes versus autosomes. We asked a question about the relative rate of interspecies sequence divergence of the X chromosome. Faster-X evolution was proposed to occur due to selection of recessive or partially recessive mutations being more efficient on the $\mathrm{X}$ chromosome than on the autosomes [Charlesworth et al., 1987]. We tested whether $\mathrm{X}$-linked genes accumulate more substitutions than autosomal genes by calculating the $\mathrm{Ka} / \mathrm{Ks}$ ratio and using $D$. grimshawi as an outgroup. The average synonymoussubstitution rate Ks values of $\mathrm{X}$-linked genes were greater than those of autosomal genes in 2 out of 3 species with the exception of D. heteroneura (Fig. 4). As Ks is usually considered to reflect nonadaptive changes, a greater Ks of $\mathrm{X}$-linked genes is expected due to the smaller effective population size $(\mathrm{Ne})$ of $\mathrm{X}$-linked relative to autosomal genes. The $\mathrm{Ka} / \mathrm{Ks}$ values were also greater in $\mathrm{X}$-linked genes compared to autosomal genes in D. planitibia (Mann-Whitney-Wilcoxon test, $p=0.049$ ) and D. silvestris $(p=0.804)$, overall consistent with the faster-X chromosome divergence. However, more robust approaches, such as McDonald-Kreitman tests, will be required to further examine this phenomenon in Hawaiian Drosophila species, based on multiple individuals being sequenced for each species.

\section{Discussion}

LCM is a powerful technique that permits rapid dissection and isolation of tissues, cells, organelles, chromosomes, and their fragments. It allows accurate investigation of subcellular or tissue-specific profiles, including disease-associated ones, especially when combined with PCR amplification, gene expression assays [EmmertBuck et al., 1996; Nakazono et al., 2003], proteomic analysis [Xu et al., 2002], enzyme recovery from LCM-transferred tissue [Emmert-Buck et al., 1996], or even profiling at the single-cell level [Kamme et al., 2004; Keays et al., 2005]. The proposed approach of mapping scaffolds to chromosomes using LCM is a fast and cost-efficient way for acquiring chromosomal information for draft genome assemblies, avoiding likely errors of reference- or synteny-based approaches. The LCM-based approach enables rapid downstream chromosome-related analyses, essentially along with the de novo genome assembly construction, and it can aid in chromosome mapping that integrates genetic and physical maps by pre-classifying scaffolds into different categories.

We exemplify the utility of the approach in application to a test for faster-X chromosome evolution [Charles- 
worth et al., 1987]. Although the X chromosome is usually similar to the autosomes in size and cytogenetic appearance, theoretical models predict that the hemizygosity of males may cause unusual patterns of evolution in the X chromosome [Vicoso and Charlesworth, 2006]. Tests for faster-X chromosome evolution in Drosophila and mammals have been largely inconclusive, necessitating more research [Meisel and Connallon, 2013]. Early studies of the faster-X evolution in coding sequences of Drosophila species led to contradictory results [Betancourt et al., 2002; Thornton and Long, 2002; Thornton et al., 2006], but increasingly more studies tend to support this phenomenon [Charlesworth and Campos, 2014; Veeramah et al., 2014]. Newer studies also include a wider taxonomical spectrum, and include human, mouse, and birds [Kousathanas et al., 2014; Wang et al., 2014; Dean et al., 2015; Wright et al., 2017]. In addition to sequence divergence, divergent gene expression patterns in Drosophila and mammals are also pronounced among Xlinked genes [Hu et al., 2013; Kousathanas et al., 2014]. The analysis of the relative divergence rates between Xlinked genes and autosomal genes in Hawaiian Drosoph$i l a$, a group that has undergone rapid adaptive radiation, is thus of special interest. In 2 of the 3 species (D. planitibia and $D$. silvestris), $\mathrm{Ka} / \mathrm{Ks}$ ratios were higher among $\mathrm{X}$-linked genes. $\mathrm{Ka} / \mathrm{Ks}$ estimates in the third species, $D$. heteroneura, are likely affected by drift effects, for this is a species with smaller effective population size [Kang et al., 2016]. Further effort to sequence and analyze other species of Hawaiian Drosophila is evidently needed to confirm this pattern in a broader and statistically more rigorous framework.

Caution is recommended when the LCM-based method is applied to the detection of structural chromosome changes, such as translocations, given the relatively high rate of DNA contamination we observed. Cross-chromo- some contamination may occur during microdissections. Therefore, care should be taken when dissecting a chromosome if material of another chromosome is nearby. The unwanted chromosomal material should be removed by the laser before proceeding to dissection of the target chromosome. Also, a misidentified chromosome arm or unnoticed attached fragment of another chromosome could create the pattern in which materials from different chromosomes are in the same sample. For this reason, familiarity with the chromosome banding pattern can be important.

The LCM technique, combined with NGS, can be applied to organisms without polytene chromosomes as long as the size of the chromosomes is large enough to meet the PicoPlex kit requirement of 6-60 pg of DNA input. Species with only microchromosomes may not be suitable for such studies until further progress in extraction of low-quantity DNA is made. Any species from such genera as Chironomus, Anopheles, Drosophila, Callipho$\mathrm{ra}$, and other dipterans, containing polytene chromosomes can be used in a similar study.

\section{Acknowledgements}

Ken Kaneshiro kindly provided D. differens. The work on chromosome microdissection was supported by the Fralin Life Science Institute and the USDA National Institute of Food and Agriculture Hatch project 223822 to I.S.

\section{Statement of Ethics}

The authors have no ethical conflicts to disclose.

\section{Disclosure Statement}

The authors have no conflicts of interest to declare.

\section{References}

-Aganezov S, Sitdykova N, AGC Consortium, Alekseyev MA: Scaffold assembly based on genome rearrangement analysis. Comput Biol Chem 57:46-53 (2015).

Aronesty E: Comparison of sequencing utility programs. Open Bioinform J 7:1-8 (2013).

Aulard S, Vaudin P, Ladeveze V, Chaminade N, Periquet G, Lemeunier F: Maintenance of a large pericentric inversion generated by the hobo transposable element in a transgenic line of Drosophila melanogaster. Heredity 92:151155 (2004).

LCM for Mapping Genomic Scaffolds to

Drosophila Chromosomes

\footnotetext{
Betancourt AJ, Presgraves DC, Swanson WJ: A Carson HL: Chromosomal sequences and interistest for faster X evolution in Drosophila. Mol Biol Evol 19:1816-1819 (2002).

- Brill E, Kang L, Michalak K, Michalak P, Price DK: Hybrid sterility and evolution in Hawaiian Drosophila: differential gene and allelespecific expression analysis of backcross males. Heredity 117:100-108 (2016). land colonizations in Hawaiian Drosophila. Genetics 103:465-482 (1983).

Carson HL: The Wilhelmine E. Key 1996 Invitational Lecture. Sexual selection: a driver of genetic change in Hawaiian Drosophila. J Hered 88:343-352 (1997).

Carson HL, Clague DA: Geology and biogeography of the Hawaiian Islands, in Wagner W, Funk V (eds): Hawaiian Biogeography: Evolution in a Hotspot Archipelago, pp 14-29 (Smithsonian Institution Press, Washington 1995).
}

Cytogenet Genome Res 2017;152:204-212 DOI: $10.1159 / 000481790$ 
Carson HL, Templeton AR: Genetic revolutions in relation to speciation phenomena - the founding of new populations. Annu Rev Ecol Syst 15:97-131 (1984).

Chamala S, Chanderbali AS, Der JP, Lan T, Walts $B$, et al: Assembly and validation of the genome of the nonmodel basal angiosperm Amborella. Science 342:1516-1517 (2013).

Charlesworth B, Campos JL: The relations between recombination rate and patterns of molecular variation and evolution in Drosophila. Annu Rev Genet 48:383-403 (2014).

Charlesworth B, Coyne JA, Barton NH: The relative rates of evolution of sex chromosomes and autosomes. Am Nat 130:113-146 (1987).

Dean R, Harrison PW, Wright AE, Zimmer F, Mank JE: Positive selection underlies faster- $Z$ evolution of gene expression in birds. Mol Biol Evol 32:2646-2656 (2015).

Dean RA, Talbot NJ, Ebbole DJ, Farman ML, Mitchell TK, et al: The genome sequence of the rice blast fungus Magnaporthe grisea. $\mathrm{Na}$ ture 434:980-986 (2005).

Dong Y, Xie M, Jiang Y, Xiao N, Du X, et al: Sequencing and automated whole-genome optical mapping of the genome of a domestic goat (Capra hircus). Nat Biotechnol 31:135141 (2013).

Drosophila 12 Genomes Consortium: Evolution of genes and genomes on the Drosophila phylogeny. Nature 450:203-218 (2007).

Ekblom R, Wolf JB: A field guide to whole-genome sequencing, assembly and annotation. Evol Appl 7:1026-1042 (2014).

-Emmert-Buck MR, Bonner RF, Smith PD, Chuaqui RF, Zhuang Z, et al: Laser capture microdissection. Science 274:998-1001 (1996).

Genome $10 \mathrm{~K}$ Community of Scientists: Genome $10 \mathrm{~K}$ : a proposal to obtain whole-genome sequence for 10,000 vertebrate species. J Hered 100:659-674 (2009).

George P, Sharma A, Sharakhov IV: 2D and 3D chromosome painting in malaria mosquitoes. J Vis Exp 83:e51173 (2014).

Grabherr MG, Haas BJ, Yassour M, Levin JZ, Thompson DA, et al: Full-length transcriptome assembly from RNA-Seq data without a reference genome. Nat Biotechnol 29:644652 (2011).

Holt C, Yandell M: MAKER2: an annotation pipeline and genome-database management tool for second-generation genome projects. BMC Bioinformatics 12:491 (2011).

- Hu TT, Eisen MB, Thornton KR, Andolfatto P: A second-generation assembly of the Drosophila simulans genome provides new insights into patterns of lineage-specific divergence. Genome Res 23:89-98 (2013).

Husemann P, Stoye J: r2cat: synteny plots and comparative assembly. Bioinformatics 26: 570-571 (2010).

i5K Consortium: The i5K Initiative: advancing arthropod genomics for knowledge, human health, agriculture, and the environment. J Hered 104:595-600 (2013).
Kamme F, Zhu J, Luo L, Yu J, Tran DT, et al: Single-cell laser-capture microdissection and RNA amplification. Methods Mol Med 99: 215-223 (2004).

Kang L, Settlage R, McMahon W, Michalak K, Tae $\mathrm{H}$, et al: Genomic signatures of speciation in sympatric and allopatric Hawaiian picturewinged Drosophila. Genome Biol Evol 8: 1482-1488 (2016).

Keays KM, Owens GP, Ritchie AM, Gilden DH, Burgoon MP: Laser capture microdissection and single-cell RT-PCR without RNA purification. J Immunol Methods 302:90-98 (2005).

Kim J, Larkin DM, Cai Q, Asan, Zhang Y, et al: Reference-assisted chromosome assembly. Proc Natl Acad Sci USA 110:1785-1790 (2013).

Korf I: Gene finding in novel genomes. BMC Bioinformatics 5:59 (2004).

Kousathanas A, Halligan DL, Keightley PD: Faster-X adaptive protein evolution in house mice. Genetics 196:1131-1143 (2014).

Li H, Durbin R: Fast and accurate short read alignment with Burrows-Wheeler transform. Bioinformatics 25:1754-1760 (2009).

Magnacca KN, Price DK: Rapid adaptive radiation and host plant conservation in the $\mathrm{Ha}$ waiian picture wing Drosophila (Diptera: Drosophilidae). Mol Phylogenet Evol 92:226242 (2015).

Mayr E: Animal Species and Evolution (Belknap Press of Harvard University Press, Cambridge 1963).

Mayr E: The Growth of Biological Thought: Diversity, Evolution, and Inheritance (Belknap Press of Harvard University Press, Cambridge 1982).

Meisel RP, Connallon T: The faster-X effect: integrating theory and data. Trends Genet 29: 537-544 (2013).

Nakazono M, Qiu F, Borsuk LA, Schnable PS: Laser-capture microdissection, a tool for the global analysis of gene expression in specific plant cell types: identification of genes expressed differentially in epidermal cells or vascular tissues of maize. Plant Cell 15:583596 (2003).

Nielsen R, Yang Z: Likelihood models for detecting positively selected amino acid sites and applications to the HIV-1 envelope gene. Genetics 148:929-936 (1998).

- Obbard DJ, Maclennan J, Kim KW, Rambaut A, O'Grady PM, Jiggins FM: Estimating divergence dates and substitution rates in the Drosophila phylogeny. Mol Biol Evol 29:34593473 (2012).

O'Grady PM, Lapoint RT, Bonacum J, Lasola J, Owen E, et al: Phylogenetic and ecological relationships of the Hawaiian Drosophila inferred by mitochondrial DNA analysis. Mol Phylogenet Evol 58:244-256 (2011).

Parra G, Bradnam K, Korf I: CEGMA: a pipeline to accurately annotate core genes in eukaryotic genomes. Bioinformatics 23:1061-1067 (2007).
Schaeffer SW, Bhutkar A, McAllister BF, Matsuda M, Matzkin LM, et al: Polytene chromosomal maps of 11 Drosophila species: the order of genomic scaffolds inferred from genetic and physical maps. Genetics 179:1601-1655 (2008). Schlötterer C, Kofler R, Versace E, Tobler R, Franssen SU: Combining experimental evolution with next-generation sequencing: a powerful tool to study adaptation from standing genetic variation. Heredity 114:431-440 (2015).

Sievers F, Wilm A, Dineen D, Gibson TJ, Karplus $\mathrm{K}$, et al: Fast, scalable generation of high-quality protein multiple sequence alignments using Clustal Omega. Mol Syst Biol 7:539 (2011).

Simão FA, Waterhouse RM, Ioannidis P, Kriventseva EV, Zdobnov EM: BUSCO: assessing genome assembly and annotation completeness with single-copy orthologs. Bioinformatics 31:3210-3212 (2015).

Stanke M, Waack S: Gene prediction with a hidden Markov model and a new intron submodel. Bioinformatics 19 Suppl 2:ii215-225 (2003).

Stark A, Lin MF, Kheradpour P, Pedersen JS, Parts L, et al: Discovery of functional elements in 12 Drosophila genomes using evolutionary

signatures. Nature 450:219-232 (2007).
Sturtevant AH, Plunkett CR: Sequence of corresponding third chromosome genes in Drosophila melanogaster and Drosophila simulans. Biol Bull 50:56-60 (1926).

Thornton K, Long M: Rapid divergence of gene duplicates on the Drosophila melanogaster X chromosome. Mol Biol Evol 19:918-925 (2002).

Thornton K, Bachtrog D, Andolfatto P: X chromosomes and autosomes evolve at similar rates in Drosophila: no evidence for faster-X protein evolution. Genome Res 16:498-504 (2006).

Veeramah KR, Gutenkunst RN, Woerner AE, Watkins JC, Hammer MF: Evidence for increased levels of positive and negative selection on the $\mathrm{X}$ chromosome versus autosomes in humans. Mol Biol Evol 31:2267-2282 (2014).

Vicoso B, Charlesworth B: Evolution on the X chromosome: unusual patterns and processes. Nat Rev Genet 7:645-653 (2006).

Wang B, Ekblom R, Bunikis I, Siitari H, Hoglund J: Whole genome sequencing of the black grouse (Tetrao tetrix): reference guided assembly suggests faster- $\mathrm{Z}$ and $\mathrm{MHC}$ evolution. BMC Genomics 15:180 (2014).

Wright AE, Darolti I, Bloch NI, Oostra V, Sandkam B, et al: Convergent recombination suppression suggests role of sexual selection in guppy sex chromosome formation. Nat Commun 8:14251 (2017).

Xu BJ, Caprioli RM, Sanders ME, Jensen RA: Direct analysis of laser capture microdissected cells by MALDI mass spectrometry. J Am Soc Mass Spectrom 13:1292-1297 (2002).

Yang Z: PAML 4: phylogenetic analysis by maximum likelihood. Mol Biol Evol 24:1586-1591 (2007). 